\title{
Resistive switching in $\beta-\mathrm{SrV}_{6} \mathrm{O}_{15}$
}

\author{
A. Akrap ${ }^{\mathrm{a}}$, R. Gaal, and L. Forró \\ Institut de Physique de la matière complexe, EPFL, 1015 Lausanne, Switzerland
}

Received 25 July 2007 / Received in final form 30 November 2007

Published online 22 February 2008 - (c) EDP Sciences, Società Italiana di Fisica, Springer-Verlag 2008

\begin{abstract}
We investigate the pressure and temperature behavior of current-dependent resistivity of $\beta$ - $\mathrm{SrV}_{6} \mathrm{O}_{15}$. We observe a switching between states of different resistivities in the insulating state of $\beta$ - $\mathrm{SrV}_{6} \mathrm{O}_{15}$. In the low pressure phase, the resistive switching appears at temperatures below the semiconductor-insulator transition. In the high pressure phase, under $\sim 1.6 \mathrm{GPa}$, the switching appears in the temperature range of the phase transition. The existence of switching may imply an important role of strontium off-stoichiometry for the electrical transport in $\beta-\mathrm{SrV}_{6} \mathrm{O}_{15}$. No electric-field-induced enhancement of the conductivity is observed. However, the conduction is significantly nonlinear under $\sim 1.6 \mathrm{GPa}$, indicating that the charge order pattern in the high pressure phase is considerably different from that of the low pressure phase.
\end{abstract}

PACS. 71.30. + h Metal-insulator transitions and other electronic transitions $-72.60 .+\mathrm{g}$ Mixed conductivity and conductivity transitions

The nature of the semiconductor-insulator transition in $\mathrm{SrV}_{6} \mathrm{O}_{15}$ remains an unsettled issue, despite the experimental and theoretical efforts [1-4]. This compound belongs to a family of quasi-one-dimensional $\beta$-vanadium bronzes, known as $\mathrm{AV}_{6} \mathrm{O}_{15}$ or $\mathrm{A}_{0.33} \mathrm{~V}_{2} \mathrm{O}_{5}$. In these systems, a metal or semiconductor-insulator transition is present at ambient pressure for majority of intercalated monovalent $\left(\mathrm{A}^{+}\right)$and divalent $\left(\mathrm{A}^{2+}\right)$ cations [5]. While this instability was first attributed to a charge ordering of vanadium atoms into $V^{4+}$ and $V^{5+}$ states [1], the picture was later changed in favor of the establishment of a long range ordered modulation of charge density: a charge density wave (CDW) $[6,7]$. The phase diagram is additionally enriched at higher pressures, where systems with monovalent cations become superconducting, whereas no superconductivity appears in divalent cation compounds $[2,8]$. The reason for the presence of a superconducting phase in $\mathrm{A}^{+}$compounds and its absence in $\mathrm{A}^{2+}$ compounds is presently not resolved either.

The physical properties of both a charge ordered and a CDW phase may a priori depend on the magnitude of the applied electric field. Charge ordering may lead to a coexistence of different phases, which generically causes a sensitivity of physical properties to external perturbations, such as electric or magnetic field $[9,10]$. Similarly, a CDW may show electric-field-dependent conductivity, and a high enough field may eventually depin the CDW modulation from the underlying lattice $[11,12]$. This should then lead to a coherent charge transport and a strong and sudden decrease in the resistivity. In case of a strong com-

\footnotetext{
a e-mail: ana.akrap@epfl.ch
}

mensurability pinning of the CDW to the lattice, such an effect would be considerably more difficult to observe. Subsequently, the two possible ground states, charge order and CDW, are expected to respond differently to the excitation current. The field-dependent conductivity measurements which were performed on $\mathrm{NaV}_{6} \mathrm{O}_{15}$ [7] showed behavior which the authors interpreted as being characteristic of CDW systems. The conductivity exhibited nonlinearity at a very low field of $0.06 \mathrm{mV} / \mathrm{cm}$, and the value of the threshold field, above which the conductivity strongly increases, was determined to be $30 \mathrm{mV} / \mathrm{cm}$. Both of the values are several orders of magnitude smaller than the corresponding quantities in the classical CDW compounds [11].

To verify if similar nonlinearities in conduction occur in $\mathrm{SrV}_{6} \mathrm{O}_{15}$, we have performed a series of $I-V$ scans in a broad temperature range. Since this compound shows remarkable sensitivity to applied pressure, we have introduced the pressure as an additional parameter. Our main finding is that there is a switching from lower to higher resistivity states which takes place at rather low excitation currents. The resistive switching may be described by two limiting values of the electric field in the sample, which are independent of either pressure or temperature. At lower pressures, the highest temperature where the switching occurs is significantly below the semiconductor-insulator phase transition. At the highest pressure we applied, the switching happens also above the phase transition. Our results favor an interpretation of the transition in terms of charge ordering. Moreover, they suggest the presence of extra electrons, which are localized in the vicinity of the excess strontium atoms. A sufficiently high electric field 


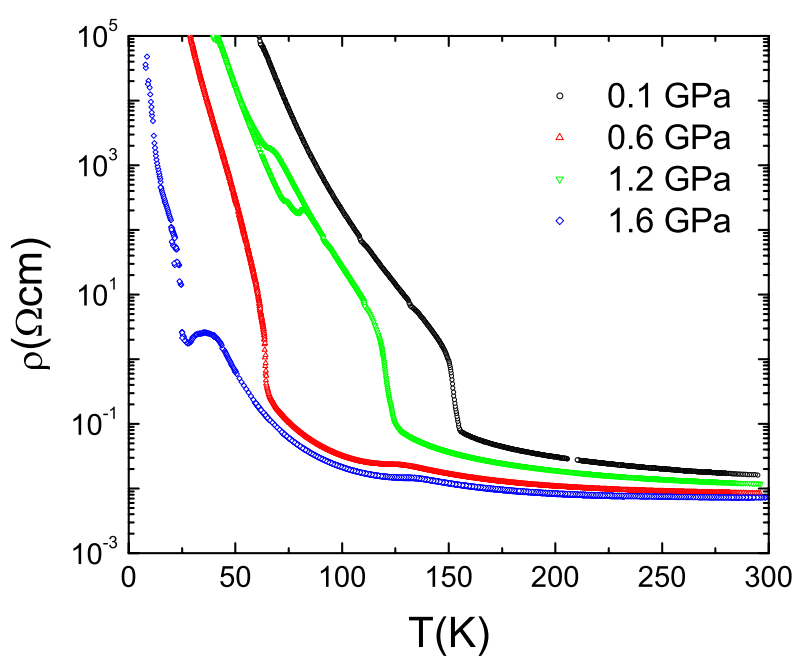

Fig. 1. The temperature dependence of the resistivity along the chain direction, at pressures where nonlinearity was investigated. All the resistivity curves, except the one for 1.6 GPa, were taken at a high bias. At $1.6 \mathrm{GPa}$, the low-temperature response to the current excitation is nonlinear in the whole accessible range of currents.

applied to the sample in the insulating phase may stretch the charge ordered pattern and localize these electrons within it.

Single crystals of $\beta-\mathrm{SrV}_{6} \mathrm{O}_{15}$ were grown as described in the work of Sellier et al. [13]. To determine the $I-V$ characteristics, we used a needle-shaped crystal of dimensions $0.5 \times 0.08 \times 0.047 \mathrm{~mm}^{3}$. The sample was equipped with four silver paint contacts, and the measurement was performed by changing the current excitation and recording the voltage. The distance between the voltage contacts was $0.33 \mathrm{~mm}$. We performed the measurements of voltage across the sample using both increasing and decreasing current. For the high electric field measurements, current was applied in short pulses in order to eliminate the effect of Joule heating. The pressure medium used was kerosene, the maximum pressure was 1.6 GPa. The pressure was determined by a calibrated InSb pressure gauge. The experimental results were confirmed on another single crystal of $\mathrm{SrV}_{6} \mathrm{O}_{15}$.

The temperature dependence of the resistivity in the relevant pressure range is shown in Figure 1. Above the phase transition at $T_{\mathrm{c}}=155 \mathrm{~K}$, the resistivity at $0.1 \mathrm{GPa}$ may approximately be described by an activated behavior formula, $\rho(T)=\rho_{0} \exp \left(\Delta_{H T} / k_{B} T\right)$. This gives an energy gap of $\Delta_{H T} \sim 430 \mathrm{~K}$. Below $T_{\mathrm{c}}$, the resistivity sharply increases and a larger gap of $\Delta_{L T} \sim 1100 \mathrm{~K}$ opens up. The transition is followed by a minute increase in the spin susceptibility [1] and is accompanied by a threefold lattice modulation [13]. Only under $T \sim 50 \mathrm{~K}$ does the susceptibility drop, indicating that the ground state is spin gapped.

The phase transition strongly depends on the pressure. The $T_{\mathrm{c}}$ quickly shifts to lower temperatures, and the nature of the transition changes from second to first order, as it is discussed elsewhere [14]. Such a decrease of

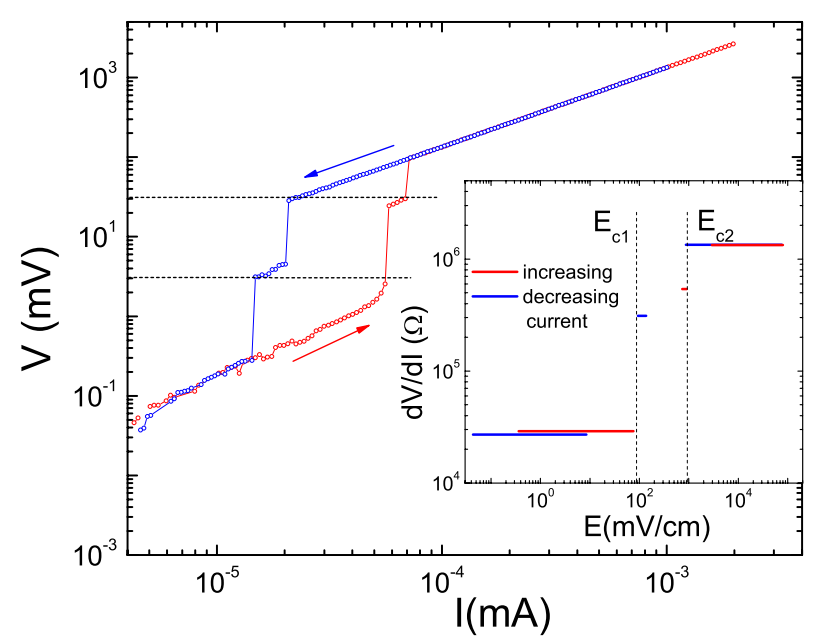

Fig. 2. The dependence of the induced voltage on the excitation current shown for an increasing current (red) and a decreasing current (blue), at $85 \mathrm{~K}$. The pressure is $\sim 0.1 \mathrm{GPa}$. The inset shows the corresponding differential resistivity, $d V / d I$, with respect to the electrical field. It was calculated from linear fits in the continuous parts. In both the main panel and the inset, the voltages/electric fields where switching takes place are marked by vertical dotted lines.

the $T_{\mathrm{c}}$ would, in a CDW picture, be the consequence of a pressure-induced change in the commensurability or the nesting conditions. Therefore it may be expected that the pressure could strongly influence the threshold field. Indeed, at the highest pressure applied in this study, the resistivity shows a remarkable sensitivity to the applied measuring current when the temperature drops below $40 \mathrm{~K}$. Accordingly, the highest pressure curve in the Figure 1 cannot give a good definition of the $T_{\mathrm{c}}$. In fact, a sharp transition from a lower to a higher resistivity may take place in a $20 \mathrm{~K}$ wide temperature range, depending on the excitation current applied.

At the lowest applied pressure, $0.1 \mathrm{GPa}$, no nonlinearity in the conduction is observed above the temperature of the phase transition $\left(T_{\mathrm{c}} \approx 155 \mathrm{~K}\right)$, even up to electrical fields as high as $150 \mathrm{~V} / \mathrm{cm}$ (not shown). This is in contrast with the measurements performed on $\mathrm{NaV}_{6} \mathrm{O}_{15}$, where the nonlinear response to the electric field seems to persist in the metallic phase [7]. However, in the insulating phase, below $130 \mathrm{~K}$, at a rather low electrical field of $91 \mathrm{mV} / \mathrm{cm}$, we could consistently observe the switching in resistivity. As the temperature is further decreased, the switching behavior persists, and the corresponding jump in the resistivity increases.

A typical $I-V$ curve displaying most of the features of the resistive switching in $\mathrm{SrV}_{6} \mathrm{O}_{15}$ is shown in Figure 2. It corresponds to the resistivity profile at $85 \mathrm{~K}$, which pertains to the insulating state - approximately $70 \mathrm{~K}$ below the phase transition. The two curves, representing the increasing and decreasing current, form a closed hysteretic loop with a feature of double threshold biases. As it is shown in the inset of Figure 2, this behavior is equivalent to a switching between a low-resistive and a highresistive state, passing through a meta-stable state of an 


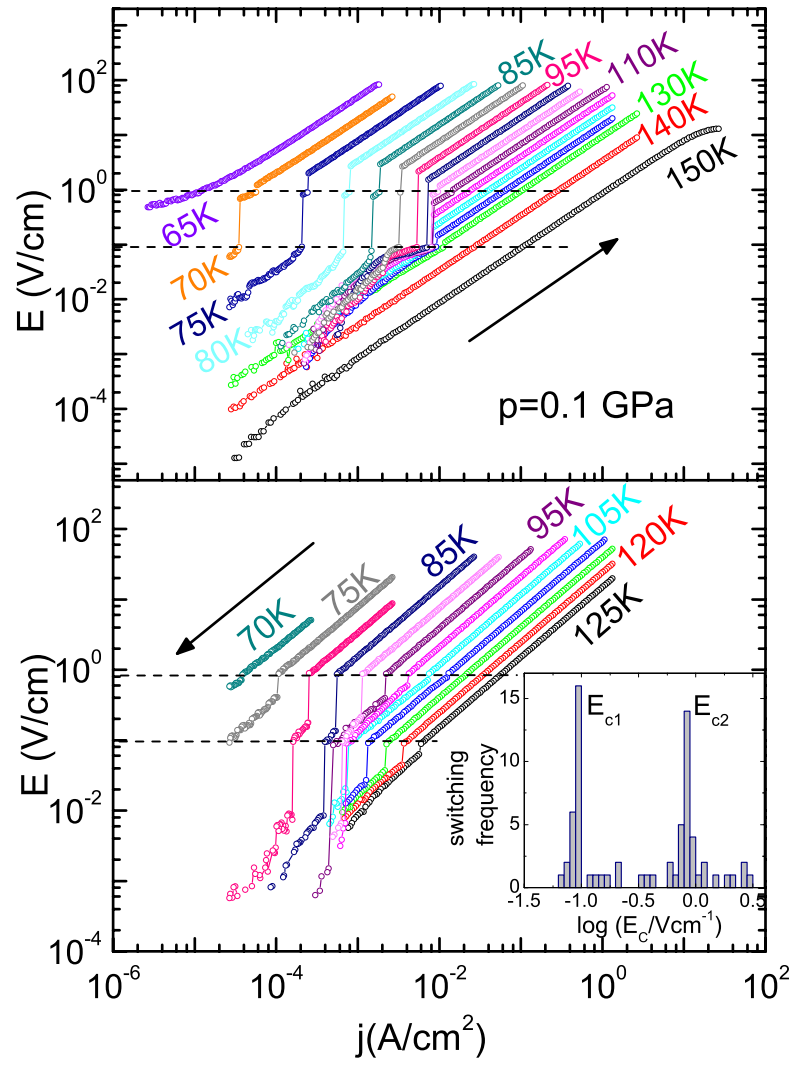

Fig. 3. The dependence of the induced voltage on the excitation current shown for an increasing current (top panel) and a decreasing current (bottom panel), under 0.1 GPa. The curves were taken at a series of different temperatures, in steps of $5 \mathrm{~K}$ (unless indicated otherwise). Vertical broken lines mark the electric fields $E_{c 1}$ and $E_{c 2}$, which delimit the high and low resistivity phases, as described in the text. The inset shows a histogram of the electric fields which mark the abrupt jumps in the $E-j$ curves. Fields $E_{c 1}$ and $E_{c 2}$ correspond to the two distinct peaks.

intermediate resistivity. Two clear breaks in both $I-V$ curves happen at fixed voltages $V_{c 1} \approx 3 \mathrm{mV}$ and $V_{c 2} \approx$ $30 \mathrm{mV}$, which, taking into account the sample geometry, give two critical values for the field across the sample: $E_{\mathrm{c} 1} \approx 91 \mathrm{mV} / \mathrm{cm}$ and $E_{\mathrm{c} 2} \approx 910 \mathrm{mV} / \mathrm{cm}$. The meaning of the fields $E_{c 1}$ and $E_{c 2}$ is the following. $E_{c 1}$ represents the highest electrical field which can be applied to the sample and still keep it in the low-resistivity state. Analogously, $E_{c 2}$ is the lowest field which, when applied to the sample, is sufficient to keep it in the high-resistivity state.

There is an order of magnitude difference between the differential resistances $d V / d I$ of the low- and high-resistive states. The current density as small as $\sim 0.01 \mu \mathrm{A} / \mathrm{cm}^{2}$ is sufficient to cause switching between these two states. We note that, in the low pressure range, in all the regions where the $I-V$ curve is continuous, the conductivity is ohmic. Even at low temperatures, all of the observed excursions from linear behavior at electrical fields up to $\sim 150 \mathrm{~V} / \mathrm{cm}$ are caused exclusively by Joule heating due to the current flow. This was verified by applying short

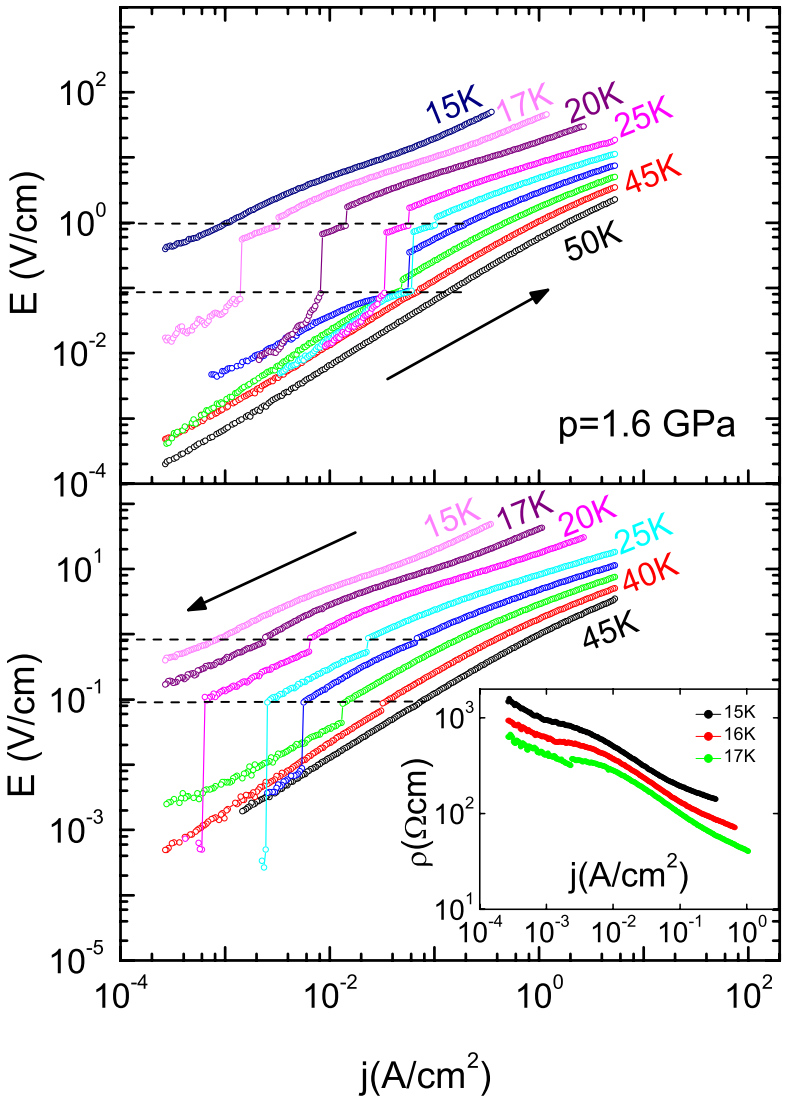

Fig. 4. The dependence of the induced electric field on the density of excitation current is shown for an increasing current (upper panel) and a decreasing current (lower panel). The curves were taken at a series of temperatures, in steps of $5 \mathrm{~K}$ (unless indicated otherwise). The applied pressure was 1.6 GPa. Vertical broken lines denote the values of electric field at which the switching takes place. The inset of the lower panel shows how the resistivity varies with current density in the low temperature phase.

current pulses, in which case the $I-V$ curves were linear. Hence, we observed no depinning of the charge distribution, in contrast to the reported coherent transport in $\mathrm{NaV}_{6} \mathrm{O}_{15}$ [7].

Since the pressure has a large impact on the physics of $\mathrm{SrV}_{6} \mathrm{O}_{15}$, it is interesting to see whether it also influences the switching behavior. Figures 3 and 4 show the dependence of the electric field in the sample on the excitation current density, for various temperatures and under two different pressures, 0.1 GPa and 1.6 GPa. A common feature across the board is that the fields $E_{\mathrm{c} 1}$ and $E_{\mathrm{c} 2}$ seem to not to depend either on temperature or on pressure. This would be very unlikely to happen in case of a CDW instability, where one would expect a significant pressure dependence of the threshold field.

In Figure 3, the temperature evolution of switching is shown for the low pressure phase. As the system is cooled, the switching first appears at $130 \mathrm{~K}$, when the phase transition has already taken place and the system is in the insulating phase. From $130 \mathrm{~K}$ down to $100 \mathrm{~K}$ the resistivity 


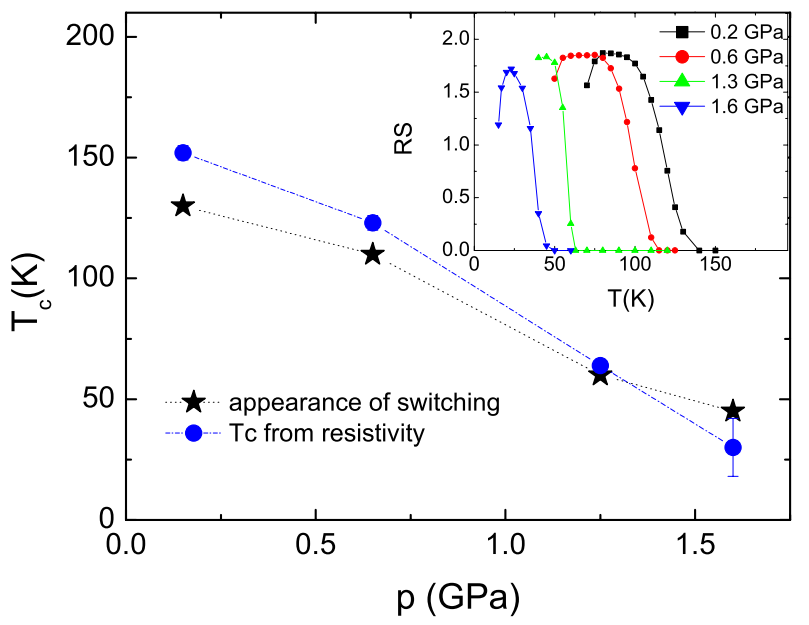

Fig. 5. The appearance of switching behavior in relation to the temperature of the phase transition. The inset shows the temperature dependence of the factor RS (defined in the text) at different pressures.

changes abruptly only at the field $E_{\mathrm{c} 1}$. Below $100 \mathrm{~K}$, another threshold field develops at $E_{\mathrm{c} 2}$. The low-resistivity state seems to exhibit a nonlinearity in the conduction, in the temperature range from 90 to $110 \mathrm{~K}$.

Under high pressure, as shown in Figure 4, the switching takes place for an order of magnitude larger current densities, $0.1 \mu \mathrm{A} / \mathrm{cm}^{2}$. At variance with the low pressure conduction, the differential resistivity is markedly nonlinear even in the continuous parts, which can be seen from the inset in Figure 4. The nonlinearity is particularly evident at low temperatures, and may be interpreted as an indication that the high-pressure insulating phase differs from the low-pressure one. Such deformations of charge modulation caused by an electric field are also observed in some of the CDW systems, and are attributed to the stretching of the localized charge modulation [15].

A switching behavior, similar to what is shown in Figures 3 and 4 , is observed in the intermediate pressure range, at 0.6 and $1.2 \mathrm{GPa}$ (not shown). At those pressures, the $E-j$ curves resemble the $0.1 \mathrm{GPa}$ ones. Namely, the continuous parts of the curves are linear, unlike those taken at 1.6 GPa, which show pronounced excursion from such simple behavior (Fig. 3). Some general trends of the resistive switching in $\mathrm{SrV}_{6} \mathrm{O}_{15}$ are displayed in Figure 5 . As the applied pressure increases, the highest temperature where the switching occurs, approaches the temperature of the phase transition, $T_{\mathrm{c}}$. Interestingly, under 1.6 GPa the switching happens already above the phase transition, which makes the resistivity curves strongly dependent on the excitation current used for the measurement. It is possible that this may be a reason for the smearing out of the phase transition at pressures higher than those we reached in the present study [16].

The magnitude of the resistance switching may be quantified by introducing the following factor [10]:

$$
R S=\frac{R_{H R}-R_{L R}}{R_{\text {average }}}=2 \frac{R_{H R}-R_{L R}}{R_{H R}+R_{L R}},
$$

where $R_{H R}$ and $R_{L R}$ are the high and low resistance values, between the two of which the switching takes place. The temperature dependence of $R S$ is shown in the inset of of Figure 5, for several pressures. The maximum value of $R S$ is $\sim 1.8$ and it is reached in the insulating phase. Such a behavior is at odds with what is observed in manganites [10], where the maximum in $R S$ occurs in a region where the resistivity is minimal and metallic. $R S$ seems to have a wider maximum in the low pressure phase, $p \lesssim 0.6 \mathrm{GPa}$, than under the highest pressure of $1.6 \mathrm{GPa}$. However, the maximal value of $R S$ does not appear to depend significantly on pressure.

A simple picture, generally compatible with charge disproportionation, emerges naturally from the above experimental observations. We may suppose that the conduction channel is inhomogeneous and consists of parts characterized by low and high resistivities. A local electric field may perturb the coexistence of phases of different electronic densities and change the relative volume of these fractions. Indeed, resistive switching was recently reported in such a system: a prototypical ternary oxide $\mathrm{SrTiO}_{3}$ [18]. The authors have demonstrated that the switching behavior is an intrinsic feature of single crystals of $\mathrm{SrTiO}_{3}$ and that it is related to the naturally occurring dislocations. They have shown that oxygen transport along filaments based on dislocations causes bistable resistive switching.

To gain further insight into the possible mechanisms of switching in $\mathrm{SrV}_{6} \mathrm{O}_{15}$, one should consider the electronic structure of the compound. The electronic system of $\mathrm{SrV}_{6} \mathrm{O}_{15}$ has a tendency to disproportionation [19], originating from the highly polarizable $\mathrm{V}_{2} \mathrm{O}_{5}$ skeleton in which the vanadium atoms have three different oxygen surroundings. As a consequence, the $d$-electrons may form some sort of a Wigner crystal already in the high-temperature phase. However, a realistic $x=1$ compound can never be perfectly stoichiometric. We may assume that there is a small surplus of strontium atoms, whose valence electrons stay localized in their vicinity. These electrons may have a role in the conduction, and in order to conduct electricity, they have to be thermally activated. On the whole, the system behaves like a lightly doped semiconductor. When the charge ordering phase transition takes place $[2,17]$, these extra electrons are still available for conduction. However, if one applies a sufficiently high electric field, the charge ordered structure will stretch to accommodate the extra electrons. When they are localized, the resistivity of the system suddenly increases. Such an effect is reversible, since decreasing the electric field releases the excess electrons and brings the system back into the more conducting state. Accordingly, there are two well-defined electric fields where the resistance switching happens.

Another possibility would be that the external electric field may actually cause the strontium atoms to move within the lattice. Again, the existence of a slight offstoichiometry is the key ingredient to understand the switching. In such a scenario, the minimum of free energy of an excess strontium atom in zero electric field corresponds to one position, but in a sufficiently high field another local minimum is accessible to the system. 
Therefore, the position of an excess strontium atom would depend on the external electric field: for a sufficiently high electric field, the excess strontium atoms jump to a new position. Presumably, the two strontium positions have different charges. When an atom jumps from out of the zero-field position, the doping of the conduction bands of the system is modified. This in turn leads to a sudden change in the conductivity. The scenario describes a phase transition of the first order in electric field. Both of the above simple pictures are compatible with the observed pressure independence of the switching fields, $E_{\mathrm{c} 1}$ and $E_{\mathrm{c} 2}$, because the pressure cannot modify the strontium off-stoichiometry.

Additional point to consider is the occurrence of a field-dependent resistivity at low temperatures under 1.6 GPa, even in the absence of switching. The present results may be interpreted in view of the recent work by Yamauchi et al. [17]. Their findings from the x-ray oscillation photographs suggest that there is a fundamental difference between the low and high pressure ground states. For $p \lesssim 1.2 \mathrm{GPa}$, they observed $1 / 2 b^{*}$, and $1 / 6 b^{*}$ or $1 / 10 b^{*}$ reflections, with $b$ being the lattice constant along the chain direction. The $1 / 6 b^{*}$ superspots were seen below $\sim 0.4 \mathrm{GPa}$, and above that pressure they were replaced by the $1 / 10 b^{*}$ superspots. However, above $p \gtrsim 1.2$ GPa only the $1 / 2 b^{*}$ reflections were seen. The latter originate from the strontium sublattice ordering, while the low-pressure $1 / 6 b^{*}$ and $1 / 10 b^{*}$ reflections are attributed to the charge ordering. Therefore, in their picture the high pressure phase ground state is charge disordered. Our high pressure $E-j$ curves, taken at $1.6 \mathrm{GPa}$, indicate that the charge distribution at low temperatures is indeed much less rigid than in the low pressure phase. Such a soft charge modulation, easily deformed by an electric field, may be caused by the absence of lattice deformation.

The existence of a lower resistivity state stabilized at low electric fields may have relevant implications for the reported measurements of magnetic susceptibility [17], since the latter does not probe the same state as the highbias resistivity. Additionally, an interesting question is whether the coexistence of states of different resistivities, observed in $\mathrm{SrV}_{6} \mathrm{O}_{15}$ but not in $\mathrm{NaV}_{6} \mathrm{O}_{15}$, could have its share in the absence of the superconductivity under higher pressures.

In conclusion, we find that in $\beta-\mathrm{SrV}_{6} \mathrm{O}_{15}$ an electrical current, and therefore also a static electric field, can trigger the collapse of a lower resistivity state to a state of high resistivity. This is observed in a wide temperature and pressure range, for pressure and temperature independent critical electrical fields. The absence of both a high-field coherent transport and a pressure dependence of the threshold fields indicates that the low temperature state of the system cannot be described by a CDW. In a more plausible picture, the electric field slightly deforms the charge ordered state in order to localize the electrons coming from the excess strontium atoms. In this way the resistivity exhibits a sudden increase for a certain value of the electric field. Moreover, the nonlinearity of the conduction at 1.6 GPa indicates that the high pressure ground state has a charge modulation different from the low-pressure charge order.

The authors are very grateful to C. Sellier, B. Corraze and E. Janod for providing well-characterized high-quality single crystals. It is a pleasure to thank S. Brazovskii for an insightful discussion, and E. Tutiš for the useful comments. This work was in part sponsored by the Swiss National Science Foundation through the NCCR pool MaNEP.

\section{References}

1. Y. Ueda, H. Yamada, M. Isobe, T. Yamauchi, J. Alloys and Compounds 317, 109 (2001)

2. T. Yamauchi, M. Isobe, Y. Ueda, Solid State Sciences 7, $874(2005)$

3. V.T. Phuoc, C. Sellier, E. Janod, Phys. Rev. B 72, 035120 (2005)

4. M.L. Doublet, M.B. Lepetit, Phys. Rev. B 71, 075119 (2005)

5. T. Yamauchi, M. Isobe, Y. Ueda, J. Magn. Mag. Mater. 272, 442 (2004)

6. S. Nagai, M. Nishi, K. Kakurai, Y. Oohara, H. Yoshizawa, H. Kimura, Y. Noda, B. Grenier, T. Yamauchi, J. Yamaura, M. Isobe, Y. Ueda, K. Hirota, J. Phys. Soc. Jpn 74, 1297 (2005)

7. S. Sirbu, T. Yamauchi, Y. Ueda, P.H.M. van Loosdrecht, Eur. Phys. J. B 53, 289 (2006)

8. T. Yamauchi, Y. Ueda, N. Môri, Phys. Rev. Lett. 89, 57002 (2002)

9. R. Fors, S.I. Khartsev, A.M. Grishin, Phys. Rev. B 71, 045305 (2005)

10. M. Quintero, P. Levy, A.G. Leyva, M.J. Rozenberg, Phys. Rev. Lett. 98, 116601 (2007)

11. G. Grüner, Reviews of Modern Physics 60, 1129 (1988)

12. G. Grüner, A. Zettl, Phys. Rep. 119, 117 (1985)

13. C. Sellier, F. Boucher, E. Janod, Solid State Sci. 5, 591 (2003)

14. A. Akrap, N. Barišić, R. Gaal, L. Forró, Phys. Rev. B 76, 235111 (2007)

15. Gy. Hutiray, G. Mihály, L. Mihály 47, 121 (1983)

16. Y. Ueda, M. Isobe, T. Yamauchi, J. Phys. Chem. Sol. 63, 951 (2002)

17. T. Yamauchi, H. Ueda, J.-I. Yamaura, Y. Ueda, Phys. Rev. B 75, 014437 (2007)

18. K. Szot, W. Speier, G. Bihlmayer, R. Waser, Nature Materials 5, 312 (2006)

19. T. Waki, M. Takigawa, T. Yamauchi, J. Yamaura, H. Ueda, Y. Ueda, e-print arXiv: cond-mat/0612017 